PROCEEDINGS OF THE

AMERICAN MATHEMATICAL SOCIETY

Volume 134, Number 4, Pages 1107-1116

S 0002-9939(05)08060-3

Article electronically published on August 12, 2005

\title{
THE WIENER-IKEHARA THEOREM BY COMPLEX ANALYSIS
}

\author{
JAAP KOREVAAR
}

(Communicated by Juha M. Heinonen)

\begin{abstract}
The Tauberian theorem of Wiener and Ikehara provides the most direct way to the prime number theorem. Here it is shown how Newman's contour integration method can be adapted to establish the Wiener-Ikehara theorem. A simple special case suffices for the PNT. But what about the twin-prime problem?
\end{abstract}

\section{INTRODUCTION}

We will use complex analysis to establish some forms of the Wiener-Ikehara theorem. Theorem 1.1 is a convenient special case. It will be derived from a useful (new) auxiliary estimate in Theorem 3.1. That estimate will be applied also to establish the general Wiener-Ikehara Theorem 5.1.

Theorem 1.1. Let the Dirichlet series

$$
\sum_{n=1}^{\infty} \frac{a_{n}}{n^{z}}, \quad \text { with coefficients } a_{n} \geq 0,
$$

converge throughout the half-plane $\{\operatorname{Re} z>1\}$. The sum function $f(z)$ will be analytic in that open half-plane. Suppose now that there is a constant $A$ such that the difference

$$
g(z)=f(z)-\frac{A}{z-1}
$$

has an analytic or continuous extension to the closed half-plane $\{\operatorname{Re} z \geq 1\}$. Finally, suppose that there is a constant $C$ such that $s_{n}=\sum_{k \leq n} a_{k} \leq C n$ for all $n$. Then

$$
s_{n} \sim A n \text { as } n \rightarrow \infty, \quad \text { that is, } s_{n} / n \rightarrow A .
$$

The hypothesis $s_{n}=\mathcal{O}(n)$ facilitates the complex approach; it will be dropped later, and did not occur in the original Wiener-Ikehara theorem. The latter was motivated by the search for a simple proof of the prime number theorem (PNT):

$$
\pi(n)=\sum_{p \text { prime, } p \leq n} 1 \sim \frac{n}{\log n} \quad \text { as } n \rightarrow \infty .
$$

The Wiener-Ikehara theorem represented an important breakthrough. In earlier results for Dirichlet series, Landau, and Hardy and Littlewood, had to impose

Received by the editors April 20, 2004 and, in revised form, November 2, 2004.

2000 Mathematics Subject Classification. Primary 40E05; Secondary 11M45, 11N05, 44 A10.

Key words and phrases. Laplace transform, prime number theorem, Tauberian theory, WienerIkehara theorem.

(C)2005 American Mathematical Society Reverts to public domain 28 years from publication 
growth conditions on $g(x+i y)$ as $y \rightarrow \pm \infty$; cf. 13. (section 66). The standard proofs use a form of Wiener theory and a suitable approximate identity; see [7, [18, 3], 14, [19] (Theorem 16). Our proof is based on the technique of contour integration which Newman [16] devised for a simple proof of the PNT. Newman's approach involved Dirichlet series with bounded coefficients. His technique was applied to Laplace transforms of bounded functions by the author 9] and Zagier 20. An adjustment of the method readily proves Theorems 1.1] and 3.1], see Section 4. The general Wiener-Ikehara theorem is reduced to Theorem 3.1 in Section 5 , In Section 2 we show that Theorem 1.1 suffices for the PNT if one makes use of Chebyshev's elementary estimate

$$
\pi(n) \leq C \frac{n}{\log n} \quad(n \geq 2) .
$$

\section{From Theorem 1.1 TO THE PRIME NUMBER TheOREM}

We will use some standard results concerning the zeta function and related number theory. Background material can be found in many books; classical references are [13] and [8]; cf. also the recent books on prime numbers [17, 15].

The zeta function provides a simple illustration of Theorem 1.1. For Re $z$ greater than 1 it is defined as the sum of the Dirichlet series

$$
\zeta(z)=f_{1}(z)=\sum_{n=1}^{\infty} \frac{1}{n^{z}} ;
$$

for other values of $z$, it is defined by analytic continuation. One has

$$
g_{1}(z)=\zeta(z)-\frac{1}{z-1}=1+z \int_{1}^{\infty}([v]-v) v^{-z-1} d v \quad(\operatorname{Re} z>1) ;
$$

cf. formulas (3.1) and (3.2). Since the integral converges for $\operatorname{Re} z>0$, it follows that $g_{1}(z)$ has an analytic continuation to that half-plane. The zeta function corresponds to the special case of Theorem 1.1 with $a_{n} \equiv 1$ and $A=1$. Here it is clear that $s_{n} \sim n$ as $n \rightarrow \infty$ !

For $\operatorname{Re} z>1$ the zeta function can be represented by the Euler product:

$$
\zeta(z)=\prod_{p \text { prime }}\left(1+\frac{1}{p^{z}}+\frac{1}{p^{2 z}}+\cdots\right)=\prod_{p \text { prime }} \frac{1}{1-p^{-z}} .
$$

In particular, $\zeta(z) \neq 0$ for $\operatorname{Re} z>1$. Logarithmic differentiation of (2.3) shows that

$$
f_{2}(z) \stackrel{\text { def }}{=}-\frac{\zeta^{\prime}(z)}{\zeta(z)}=\sum_{p \text { prime }} \frac{p^{-z} \log p}{1-p^{-z}}=\sum_{p \text { prime }, m \geq 1} \frac{\log p}{p^{m z}} .
$$

Rearranging the terms one obtains the Dirichlet series

$$
f_{2}(z)=\sum_{n=1}^{\infty} \frac{\Lambda(n)}{n^{z}}
$$

where the coefficients are given by the von Mangoldt function,

$$
\Lambda(n)= \begin{cases}0 & \text { if } n=1, \\ \log p & \text { if } n=p^{m} \text { with } p \text { prime and } m \geq 1, \\ 0 & \text { if } n \text { has at least two different prime factors. }\end{cases}
$$


The partial sums $s_{n}$ of the coefficients define the Chebyshev function $\psi$ :

$$
s_{n}=\psi(n) \stackrel{\text { def }}{=} \sum_{k \leq n} \Lambda(k)=\sum_{p^{m} \leq n} \log p .
$$

By Chebyshev's inequality (1.5),

$$
\psi(n)=\sum_{p \leq n}\left[\frac{\log n}{\log p}\right] \log p \leq \pi(n) \log n \leq C n .
$$

Chebyshev knew also that the (then unproved) asymptotic relation

$$
\psi(n) \sim n \quad \text { as } n \rightarrow \infty
$$

would be equivalent to the prime number theorem.

For the application of Theorem 1.1 to $f_{2}(z)$, one has to know how this function behaves when $z$ approaches the line $\{\operatorname{Re} z=1\}$. Here one needs the crucial fact that $\zeta(z) \neq 0$ on that line. The simpler proofs of the PNT all use this nonvanishing; by Wiener's work, the PNT is equivalent to it; cf. [4, 8], 10, 11] (section III.3). As $\zeta(z) \neq 0$ on the line $\{\operatorname{Re} z=1\}$, the quotient $f_{2}(z)=-\zeta^{\prime}(z) / \zeta(z)$ is analytic there, except of course at the point $z=1$. Since $\zeta(z)$ behaves like $1 /(z-1)$ around the point $z=1$, the same is true for $f_{2}(z)$. Summarizing,

$$
g_{2}(z) \stackrel{\text { def }}{=} f_{2}(z)-\frac{1}{z-1}
$$

is analytic at every point of the closed half-plane $\{\operatorname{Re} z \geq 1\}$.

The numbers $a_{n}=\Lambda(n)$ and the corresponding functions $f=f_{2}$ and $g=g_{2}$ satisfy the conditions of Theorem 1.1 with $A=1$; the partial sums $s_{n}=\psi(n)$ are bounded by $C n$. Hence, Theorem 1.1 implies (2.9), which gives the PNT.

\section{Reduction of Theorem 1.1}

Let the conditions of Theorem 1.1 be satisfied, including the condition $s_{n}=$ $\mathcal{O}(n)$. The proof of the theorem will be derived from a result on Laplace transforms of bounded functions. Define

$$
s(v)=\sum_{k \leq v} a_{k},
$$

so that $s(v)$ is nondecreasing, $s(v)=s_{n}$ for $n \leq v<n+1$ and $s(v)=0$ for $v<1$.

Partial summation applied to the series in (1.1) shows that for $\operatorname{Re} z>1$,

$$
\begin{aligned}
f(z) & =\sum_{n=1}^{\infty} \frac{s_{n}-s_{n-1}}{n^{z}}=\sum_{n=1}^{\infty} s_{n}\left\{\frac{1}{n^{z}}-\frac{1}{(n+1)^{z}}\right\} \\
& =\sum_{n=1}^{\infty} s_{n} z \int_{n}^{n+1} v^{-z-1} d v=z \int_{1}^{\infty} s(v) v^{-z-1} d v .
\end{aligned}
$$

In view of (1.2) one can now write

$$
\begin{aligned}
g(z)-A & =f(z)-\frac{A}{z-1}-A=f(z)-\frac{A z}{z-1} \\
& =z \int_{1}^{\infty}\left\{\frac{s(v)}{v}-A\right\} v^{-z} d v .
\end{aligned}
$$


For $v \geq 1$ we finally substitute

$$
v=e^{t}, \quad \frac{s(v)}{v}-A=e^{-t} s\left(e^{t}\right)-A=\rho(t) ;
$$

we set $\rho(t)=0$ for $t<0$. Our assumptions imply that $\rho(t)$ is a bounded function, which cannot decrease rapidly as $t \rightarrow \infty$. Indeed, for $t>u \geq 0$,

$$
\begin{aligned}
\rho(t)-\rho(u) & =e^{-t} s\left(e^{t}\right)-e^{-u} s\left(e^{u}\right) \geq\left(e^{-t}-e^{-u}\right) s\left(e^{u}\right) \\
& =\left\{e^{-(t-u)}-1\right\} e^{-u} s\left(e^{u}\right)=-\left\{1-e^{-(t-u)}\right\}\{\rho(u)+A\} \\
& \geq-(t-u)\{\rho(u)+A\} .
\end{aligned}
$$

Every function $\rho$ which satisfies a relation

$$
\rho(t)-\rho(u) \geq-\eta(t, u), \quad \text { where } \eta(t, u) \rightarrow 0
$$

as $u \rightarrow \infty$ and $0<t-u \rightarrow 0$, is called slowly decreasing, although such a function might well be increasing!

We now consider the Laplace transform of $\rho$ :

$$
\begin{aligned}
\mathcal{L} \rho(z) & \stackrel{\text { def }}{=} \int_{0}^{\infty} \rho(t) e^{-z t} d t=\int_{1}^{\infty}\left\{\frac{s(v)}{v}-A\right\} v^{-z-1} d v \\
& =\frac{g(z+1)-A}{z+1} .
\end{aligned}
$$

The hypotheses of Theorem 1.1 imply that $\mathcal{L} \rho(z)$ is analytic for $\operatorname{Re} z>0$, and has an analytic or continuous extension to the half-plane $\{\operatorname{Re} z \geq 0\}$. We have to prove that $\rho(t) \rightarrow 0$ as $t \rightarrow \infty$. Thus Theorem 1.1 follows from the (more general)

Theorem 3.1. Let $\rho(t)=0$ for $t<0$ and $|\rho(t)| \leq M<\infty$ for $t \geq 0$. Then the Laplace transform

$$
G(z)=\mathcal{L} \rho(z)=\int_{0}^{\infty} \rho(t) e^{-z t} d t, \quad z=x+i y,
$$

defines an analytic function for $x>0$. Suppose that for $-R \leq y \leq R$, the function $G_{x}(i y)=G(x+i y)$ converges uniformly (or in $L^{1}$ ) to a limit function $G(i y)$ as $x \searrow 0$. Then for every positive $T$ and $\delta$,

$$
\left|\int_{T}^{T+\delta} \rho(t) d t\right| \leq \frac{4 M}{R}+\frac{1}{2 \pi}\left|\int_{-R}^{R} G(i y) \frac{e^{i \delta y}-1}{y}\left(1-\frac{y^{2}}{R^{2}}\right) e^{i T y} d y\right| .
$$

If in the hypotheses, $R$ may be taken arbitrarily large, and furthermore, the function $\rho$ is slowly decreasing, then

$$
\rho(T) \rightarrow 0 \quad \text { as } T \rightarrow \infty .
$$

Remark 3.2. Arendt, Batty, Hieber and Neubrander [1] obtained a limit relation (3.9) in their Theorem 4.9.5. Imposing somewhat different conditions, they used Fourier and functional analysis, while we use a variation on Newman's contour method [16, 9 . 


\section{Proof of Theorem 3.1}

Proof of inequality (3.8). Let $G_{T}$ be the function

$$
G_{T}(z)=\int_{0}^{T} \rho(t) e^{-z t} d t, \quad z=x+i y
$$

which is analytic in the whole complex plane. We have to estimate the difference

$$
G_{T+\delta}(0)-G_{T}(0)=\int_{T}^{T+\delta} \rho(t) d t
$$

(i) Let $\Gamma$ be the positively oriented circle $C(0, R)=\{|z|=R\}$. By Cauchy's formula,

$$
2 \pi i G_{T}(0)=\int_{\Gamma} G_{T}(z) \frac{1}{z} d z .
$$

Thinking of large $T$ and taking $z$ in the right half-plane $\{x>0\}$, it is natural to compare $G_{T}(z)$ with $G(z)$ :

$$
\left|G_{T}(z)-G(z)\right|=\left|\int_{T}^{\infty} \rho(t) e^{-z t} d t\right| \leq M \int_{T}^{\infty} e^{-x t} d t=\frac{M}{x} e^{-T x} .
$$

Somewhat analogously one finds for $x=\operatorname{Re} z<0$ that

$$
\left|G_{T}(z)\right|=\left|\int_{0}^{T} \rho(t) e^{-z t} d t\right| \leq \int_{0}^{T} M e^{-x t} d t<\frac{M}{|x|} e^{-T x} .
$$

(ii) For large $x$, the factor $e^{-T x}$ in (4.4) becomes much smaller than necessary, while in (4.5), the factor $e^{-T x}=e^{T|x|}$ may become much too large. One of Newman's clever ideas [16] was to introduce a factor $e^{T z}$ under the integral sign of a Cauchy formula such as (4.3):

$$
2 \pi i G_{T}(0)=\int_{\Gamma} G_{T}(z) e^{T z} \frac{1}{z} d z .
$$

There still is the problem of the denominators $x$ and $|x|$ in (4.4), (4.5) when $|x|$ becomes small. For that, Newman found the following ingenious solution: replace the factor $1 / z$ in the Cauchy formula by $(1 / z)+z / R^{2}$. On the circle $\Gamma$,

and by the residue theorem

$$
\frac{1}{z}+\frac{z}{R^{2}}=\frac{2 x}{R^{2}}
$$

$$
2 \pi i G_{T}(0)=\int_{\Gamma} G_{T}(z) e^{T z}\left(\frac{1}{z}+\frac{z}{R^{2}}\right) d z .
$$

(iii) Looking at (4.4), one would like to do something similar to (4.7) for $G(z)$ instead of $G_{T}(z)$. However, in general, $G(z)$ is not analytic in the left half-plane $\{x<0\}$, hence one cannot integrate $G(z) / z$ over $\Gamma$. What one can do is, apply Cauchy's theorem to $G(z) / z$, or to

$$
G(z) e^{T z}\left(\frac{1}{z}+\frac{z}{R^{2}}\right),
$$

if one integrates over a path that lies entirely in the right half-plane. We introduce some special paths of integration. Let $\Gamma_{1}$ be the part of $\Gamma$ in the right half-plane, $\Gamma_{2}$ the part of $\Gamma$ in the left half-plane. We denote by $\sigma$ the oriented segment of the imaginary axis from $i R$ to $-i R$; cf. Figure 1. Finally, for small $r>0$, we let $\sigma_{r}$ be 
the oriented segment of the line $x=r$ between the point $z_{1}=r+i \sqrt{R^{2}-r^{2}}$ of $\Gamma$ and the point $z_{2}=r-i \sqrt{R^{2}-r^{2}}$. The part of $\Gamma_{1}$ between $z_{2}$ and $z_{1}$ is called $\Gamma_{1, r}$. Then by Cauchy,

$$
0=\int_{\Gamma_{1, r}+\sigma_{r}} G(z) e^{T z}\left(\frac{1}{z}+\frac{z}{R^{2}}\right) d z
$$

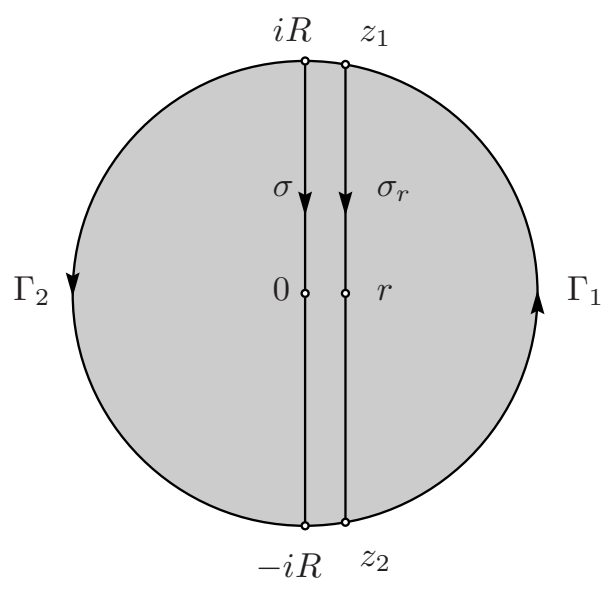

Figure 1. The paths of integration

(iv) Combining (4.7) and (4.8), one finds that for every small $r>0$,

$$
\begin{aligned}
2 \pi i G_{T}(0)= & \int_{\Gamma_{1, r}}\left\{G_{T}(z)-G(z)\right\} e^{T z}\left(\frac{1}{z}+\frac{z}{R^{2}}\right) d z \\
& +\int_{\Gamma-\Gamma_{1, r}} G_{T}(z) e^{T z}\left(\frac{1}{z}+\frac{z}{R^{2}}\right) d z \\
& -\int_{\sigma_{r}} G(z) e^{T z}\left(\frac{1}{z}+\frac{z}{R^{2}}\right) d z \\
= & I_{1}(R, r, T)+I_{2}(R, r, T)-I_{3}(R, r, T),
\end{aligned}
$$

say. There is a corresponding formula for $G_{T+\delta}(0)$. In the end, we have to estimate the difference $G_{T+\delta}(0)-G_{T}(0)$, in particular, also

$$
\begin{aligned}
I_{3}(R, r, T+\delta)-I_{3}(R, r, T) & =\int_{\sigma_{r}} G(z)\left\{e^{(T+\delta) z}-e^{T z}\right\}\left(\frac{1}{z}+\frac{z}{R^{2}}\right) d z \\
& =\int_{\sigma_{r}} G(z) \frac{e^{\delta z}-1}{z}\left(1+\frac{z^{2}}{R^{2}}\right) e^{T z} d z .
\end{aligned}
$$

By the postulated boundary behavior of $G(x+i y)$ as $x \searrow 0$, we may let $r$ go to zero here, and we may do that also in the corresponding formula for $G_{T+\delta}(0)-G_{T}(0)$. Letting $I_{j}(R, 0, \cdot)$ refer to integrals involving $\Gamma_{1}$ instead of $\Gamma_{1, r}$ and $\sigma$ instead of $\sigma_{r}$, 
we then obtain the inequality

$$
\begin{aligned}
& 2 \pi\left|G_{T+\delta}(0)-G_{T}(0)\right| \\
& \leq\left|I_{1}(R, 0, T+\delta)-I_{1}(R, 0, T)+I_{2}(R, 0, T+\delta)-I_{2}(R, 0, T)\right| \\
& \quad+\left|\int_{\sigma} G(z) \frac{e^{\delta z}-1}{z}\left(1+\frac{z^{2}}{R^{2}}\right) e^{T z} d z\right| .
\end{aligned}
$$

(v) It follows from (4.9), (4.4) and (4.6) that

$$
\begin{aligned}
\left|I_{1}(R, 0, T)\right| & \leq \int_{\Gamma_{1}}\left|G_{T}(z)-G(z)\right|\left|e^{T z}\right|\left|\frac{1}{z}+\frac{z}{R^{2}}\right||d z| \\
& \leq \int_{\Gamma_{1}} \frac{M}{x} e^{-T x} e^{T x} \frac{2 x}{R^{2}}|d z| \\
& =\frac{2 M}{R^{2}} \pi R=\frac{2 \pi M}{R} .
\end{aligned}
$$

Analogously, (4.9), (4.5) and (4.6) show that

$$
\left|I_{2}(R, 0, T)\right| \leq \int_{\Gamma_{2}}\left|G_{T}(z) e^{T z}\right|\left|\frac{1}{z}+\frac{z}{R^{2}}\right||d z| \leq \frac{2 \pi M}{R} .
$$

When $j=1,2$ we use the same inequalities for $\left|I_{j}(R, 0, T+\delta)\right|$. It is true that the differences $I_{j}(R, 0, T+\delta)-I_{j}(R, 0, T)$ are $\mathcal{O}(\delta)$ as $\delta \searrow 0$, but the constants in these estimates are not small enough to be of much help in Section 5 .

Combining (4.2) with (4.10)-(4.12) and dividing by $2 \pi$, one thus obtains

$$
\left|\int_{T}^{T+\delta} \rho(t) d t\right| \leq \frac{4 M}{R}+\frac{1}{2 \pi}\left|\int_{\sigma} G(z) \frac{e^{\delta z}-1}{z}\left(1+\frac{z^{2}}{R^{2}}\right) e^{T z} d z\right| .
$$

Substitution of $z=i y$ with $-R \leq y \leq R$ gives the desired inequality (3.8).

Proof of relation (3.9). (vi) For fixed $\delta$ and $R$, the final integral in (3.8) will go to zero as $T \rightarrow \infty$. Indeed, if $G(z)$ is analytic on the segment $[-i R, i R]$ of the imaginary axis, one can integrate by parts: $e^{i T y} d y=\{1 /(i T)\} d e^{i T y}$, etc. If one knows only that the boundary values $G(i y)$ are continuous or integrable, one can use the Riemann-Lebesgue lemma. In either case the conclusion is that

$$
\limsup _{T \rightarrow \infty}\left|\int_{T}^{T+\delta} \rho(t) d t\right| \leq \frac{4 M}{R}
$$

(vii) We now assume that $R$ may be taken arbitrarily large. Then for every number $\delta>0$,

$$
\int_{T}^{T+\delta} \rho(t) d t \rightarrow 0 \quad \text { as } T \rightarrow \infty
$$

Suppose finally that $\rho(t)$ is slowly decreasing; cf. (3.5). The inequality

$$
\int_{T}^{T+\delta}\{\rho(t)-\rho(T)\} d t \geq-\int_{T}^{T+\delta} \eta(t, T) d t
$$

and (4.15) then show that, for given $\varepsilon>0$ and sufficiently small $\delta>0$,

$$
\limsup _{T \rightarrow \infty} \delta \rho(T) \leq \varepsilon \delta, \quad \limsup _{T \rightarrow \infty} \rho(T) \leq \varepsilon
$$

This holds for every $\varepsilon>0$, so that $\lim \sup \rho(T) \leq 0$. For an inequality in the other direction, one may start with the integral $\int_{T-\delta}^{T} \rho(t) d t$. 


\section{A general Wiener-IKehara theOREM}

We consider a Wiener-Ikehara theorem for Laplace-Stieltjes transforms which contains the standard form for Dirichlet series, that is, Theorem 1.1 without the hypothesis $s_{n} \leq C n$; cf. [7], [10, [1].

Theorem 5.1. Let $S(t)$ vanish for $t<0$, be nondecreasing, continuous from the right and such that the Laplace-Stieltjes transform

$$
\begin{aligned}
f(z) & =\mathcal{L} d S(z)=\int_{0-}^{\infty} e^{-z t} d S(t) \\
& =z \int_{0}^{\infty} S(t) e^{-z t} d t=z \mathcal{L} S(z), \quad z=x+i y,
\end{aligned}
$$

exists for $\operatorname{Re} z=x>1$. Suppose that for some constant $A$, the analytic function

$$
g(z)=f(z)-\frac{A}{z-1}, \quad \operatorname{Re} z=x>1,
$$

extends analytically, continuously or in local $L^{1}$ sense to the boundary line $\{x=1\}$. Then

$$
e^{-t} S(t) \rightarrow A \quad \text { as } t \rightarrow \infty
$$

The substitutions

$$
e^{-t} S(t)-A=\rho(t), \quad G(z)=\mathcal{L} \rho(z)=\frac{g(z+1)-A}{z+1}
$$

establish a link with Theorem 3.1. Thus the proof of Theorem 5.1 can be completed by

Proposition 5.2. Let $S, f$ and $g$ be as in Theorem 5.1. Then $\rho(t)$ is bounded.

We begin with

Lemma 5.3. The function

$$
\rho_{\varepsilon}(t)=e^{-\varepsilon t} \rho(t)=e^{-(1+\varepsilon) t} S(t)-e^{-\varepsilon t} A
$$

is bounded for every number $\varepsilon>0$.

Proof. It is enough to show that $\rho_{\varepsilon}(t)$ or $e^{-(1+\varepsilon) t} S(t)$ is bounded from above. The latter follows immediately from the existence of the Laplace transform $\mathcal{L} d S(1+\varepsilon)$. Indeed, for any $T>0$,

$$
\int_{0-}^{\infty} e^{-(1+\varepsilon) t} d S(t) \geq \int_{0-}^{T} \cdots \geq e^{-(1+\varepsilon) T} S(T) .
$$

Proof of Proposition 5.2. For $\varepsilon>0$, set

$$
\sup _{t}\left|\rho_{\varepsilon}(t)\right|=\sup _{t} e^{-\varepsilon t}|\rho(t)|=M_{\varepsilon} .
$$

This is a nonincreasing function of $\varepsilon$. We may suppose that $M_{\varepsilon}$ will be larger than $S(0)+A$ for all small $\varepsilon>0$, or there is nothing to prove. By (5.5) and the properties of $S(\cdot)$, the function $\rho_{\varepsilon}(t)$ will take on the value $M_{\varepsilon}$ for some $t=T_{\varepsilon}>0$ when $\varepsilon$ is small, $\varepsilon \leq \varepsilon_{0}$, say. 
Setting $G=\mathcal{L} \rho$ as in (5.4), we now apply Theorem 3.1 to $\rho_{\varepsilon}(t)$ and its Laplace transform $G(\varepsilon+z)$. Then by inequality (3.8) with $\delta=1$ and $R>0$,

$$
\left|\int_{T}^{T+1} \rho_{\varepsilon}(t) d t\right| \leq \frac{4 M_{\varepsilon}}{R}+\frac{1}{2 \pi}\left|\int_{-R}^{R} G(\varepsilon+i y) \frac{e^{i y}-1}{y}\left(1-\frac{y^{2}}{R^{2}}\right) e^{i T y} d y\right| .
$$

Next observe that for small $\varepsilon$ and $T=T_{\varepsilon} \leq t \leq T+1$, by (5.5) and the observations following (5.6),

$$
\rho_{\varepsilon}(t) \geq e^{-(1+\varepsilon)(T+1)} S(T)-A \geq e^{-(1+\varepsilon)} \rho_{\varepsilon}(T)-A .
$$

Combination with (5.7) thus shows that for $T=T_{\varepsilon}$,

$$
e^{-1-\varepsilon} M_{\varepsilon} \leq A+\frac{4 M_{\varepsilon}}{R}+\frac{1}{2 \pi}\left|\int_{-R}^{R} G(\varepsilon+i y) \frac{e^{i y}-1}{y}\left(1-\frac{y^{2}}{R^{2}}\right) e^{i T y} d y\right| .
$$

Taking $\varepsilon \leq \varepsilon_{0} \leq 1$ and $R=8 e^{2}$, it follows that $M_{\varepsilon}$ is bounded by $2 e^{2} A$ plus $e^{2} / \pi$ times the absolute value of the integral in (5.8) with $T=T_{\varepsilon}$. Now by the boundary behavior of $G(\varepsilon+i y)$ as $\varepsilon \searrow 0$, the integral is bounded by some number $C$ independent of $\varepsilon \in\left(0, \varepsilon_{0}\right]$. It follows that

$$
M_{\varepsilon} \leq 2 e^{2} A+\left(e^{2} / \pi\right) C \text { for } 0<\varepsilon \leq \varepsilon_{0} .
$$

This bound on $M_{\varepsilon}$ implies that $\rho(\cdot)$ is bounded.

\section{Open PROBLEMS}

The boundedness proof for $\rho(\cdot)$ in Section 5 requires that $g(x+i y)$ in Theorem 5.1 have good boundary behavior as $x \searrow 1$ on a sufficiently long boundary interval. In the standard "Fourier" proof of the Wiener-Ikehara theorem, any boundary interval $\{x=1,-R \leq y \leq R\}$ suffices to establish the boundedness of $\rho(\cdot)$; cf. 10] or [11. See also the sharp "finite form" of the Wiener-Ikehara theorem in [5]. Can one modify the complex method to do as well?

[Added July 2004] A more important question involves the twin-prime conjecture (TPC) of Hardy and Littlewood [6]. It asserts that the number $\pi_{2}(n)$ of prime pairs $(p, p+2)$ with $p \leq n$ satisfies the asymptotic relation

$$
\pi_{2}(n) \sim C_{2} \frac{n}{\log ^{2} n}, \quad \text { where } C_{2}=2 \prod_{p>2}\left\{1-\frac{1}{(p-1)^{2}}\right\} .
$$

Arenstorf 2 made a serious attempt to derive (6.1) from a Wiener-Ikehara theorem. To increase the chance of success, one might use a (new) distributional WienerIkehara theorem. Trying to apply it to the Dirichlet series

$$
f_{2}^{*}(z)=\sum_{n=1}^{\infty} \frac{\Lambda(n) \Lambda(n+2)}{n^{z}}
$$

one would consider the difference

$$
g_{2}^{*}(z)=f_{2}^{*}(z)-\frac{C_{2}}{z-1}, \quad z=x+i y,
$$

and its boundary distribution $g_{2}^{*}(1+i y)$ as $x \searrow 1$. The TPC is equivalent to the assertion that $g_{2}^{*}(1+i y)$ is locally equal to a pseudofunction, that is, the Fourier transform of a bounded function which tends to zero at $\pm \infty$; see [12. 


\section{REFERENCES}

[1] W. Arendt, C.J.K. Batty, M. Hieber, F. Neubrander, Vector-Valued Laplace Transforms and Cauchy Problems, Birkhäuser, Basel, 2001. MR.1886588 (2003g:47072)

[2] R.F. Arenstorf, There are infinitely many prime twins, Preprint, Vanderbilt University, Nashville, TN; 38 pages. Posted on the arXiv:math.NT/0405509, May 26, 2004; withdrawn June 9, 2004. Available at http://arxiv.org/abs/math/0405509v1

[3] S. Bochner, Ein Satz von Landau und Ikehara, Math. Z. 37 (1933), 1-9.

[4] H.G. Diamond, Elementary methods in the study of the distribution of prime numbers, Bull. Amer. Math. Soc. (N.S.) 7 (1982), 553-589. MR0670132 (83m:10002)

[5] S.W. Graham, J.D. Vaaler, A class of extremal functions for the Fourier transform, Trans. Amer. Math. Soc. 265 (1981), 283-302. MR0607121 (82i:42008)

[6] G.H. Hardy, J.E. Littlewood, Some problems of 'partitio numerorum' III, On the expression of a number as a sum of primes, Acta Math. 44 (1922), 1-70.

[7] S. Ikehara, An extension of Landau's theorem in the analytic theory of numbers, J. Math. and Phys. 10 (1931), 1-12.

[8] A.E. Ingham, The Distribution of Prime Numbers, Cambridge Tracts in Math. no. 30, Cambridge Univ. Press, 1932. (Reprinted 1990.) MR.1074573 (91f:11064)

[9] J. Korevaar, On Newman's quick way to the prime number theorem, Math. Intelligencer 4 (1982), 108-115. MR0684025 (84b:10063)

[10] J. Korevaar, A century of complex Tauberian theory, Bull. Amer. Math. Soc. (N.S.) 39 (2002), 475-531. MR1920279 (2003g:40004)

[11] J. Korevaar, Tauberian Theory. A Century of Developments, Grundl. math. Wiss. vol. 329, Springer-Verlag, Berlin, 2004. MR:2073637

[12] J. Korevaar, Distributional Wiener-Ikehara theorem and twin primes, Indag. Math. (N.S.) 16 (2005), 37-49.

[13] E. Landau, Handbuch der Lehre von der Verteilung der Primzahlen I, II, Teubner, Leipzig, 1909. (Second edition with an appendix by P.T. Bateman, Chelsea Publ. Co., New York, 1953.) MR0068565 (16,904d)

[14] E. Landau, Über den Wienerschen neuen Weg zum Primzahlsatz, Sitz.-Ber. Preuss. Akad. Wiss., Phys.-Math. Klasse, 1932, 514-521.

[15] W. Narkiewicz, The Development of Prime Number Theory, Springer Monographs in Math., Springer-Verlag, Berlin, 2000. MR.1756780 (2001c:11098)

[16] D.J. Newman, Simple analytic proof of the prime number theorem, Amer. Math. Monthly 87 (1980), 693-696. MR0602825 (82h:10056)

[17] G. Tenenbaum, M. Mendès France, The Prime Numbers and their Distribution, Student Math. Library vol. 6, Amer. Math. Soc., Providence, RI, 2000. (Translation of the French edition of 1997.) MR,1756233 (2001j:11086)

[18] N. Wiener, Tauberian theorems, Ann. of Math. 33 (1932), 1-100. MR.1503035

[19] N. Wiener, The Fourier Integral and Certain of its Applications, Cambridge Univ. Press, Cambridge, 1933. (Reprinted 1988.) MR0983891 (90b:42022)

[20] D. Zagier, Newman's short proof of the prime number theorem, Amer. Math. Monthly 104 (1997), 705-708. MR:1476753 (98j:11069)

KdV Institute of Mathematics, University of Amsterdam, Plantage Muidergracht 24, 1018 TV Amsterdam, Netherlands

E-mail address: korevaar@science.uva.nl 